\title{
Pituitary Enhancement of Wolffian Lens Regeneration In Vitro: Spatial and Temporal Requirements ${ }^{1}$
}

\author{
THOMAS G. CONNELLY \\ 2 Department of Anatomy and Center for Human Growth and Development, University of \\ Michigan, Ann Arbor, Michigan 48109
}

\begin{abstract}
When irido-corneal complexes (ICC's) from eyes of adult newts (Notophthalmus viridescens) are cultured on top of pituitary glands they produce advanced lens regenerates in up to $90 \%$ of the cases (this report and Connelly et al., '73). To determine if the iris and pituitary gland must be in direct contact with each other in order to produce this enhancement of lens regeneration, ICC's were cultured next to pituitary glands or separated from them by a Nuclepore filter. Cultures behaved as ICC's cultured in the absence of the pituitary gland and produced few advanced lens regenerates. To determine how long the iris and pituitary must be in contact for the enhancement to occur, ICC's were explanted and 5,10 or 15 days later pituitary glands were placed beneath the dorsal iris pupillary margin. There was a progressive decrease in the number of advanced lens regenerates formed in cultures receiving pituitary glands later than five days after initial explantation of the iris. The conclusions drawn from these experiments are that the iris and pituitary gland must be in extensive direct contact during the first five to ten days in culture in order for pronounced enhancement of lens regeneration to occur.
\end{abstract}

Removal of the pituitary from an adult newt retards but does not prohibit lens regeneration in vivo (Schotte and Murphy, '53; Stone and Steinitz, '53; Connelly et al., '73). Combining the iris and the pituitary gland in organ culture results in a marked enhancement of lens regeneration in vitro (Connelly et al., '73). Other tissues and organs have been cultured with the iris, but only the neural retina has proved as effective as pituitary gland in enhancing the production of advanced lens regenerates from intact iris in vitro (Yamada et al., '73). As part of a continuing investigation of the mechanism by which the pituitary enhances lens regeneration in culture the experiments reported below were designed to deal with two questions: (1) is direct contact between the two organs necessary for enhancement to occur; and (2) when must the two organs be together during a 21-day culture period in order for enhancement of lens regeneration to result?

\section{METHODS}

Adult newts (Notophthalmus viridescens) obtained from Bill Lee Newt Farms, Oak Ridge, Tennessee were used in all experi- ments. Prior to surgery all animals were surface sterilized in a weak permanganate solution (Rounds, '73) followed by careful rinsing of the head and eyes in 70\% ethanol and sterile distilled water. All surgical manipulations, medium preparation and medium changes were carried out in a laminar airflow hood. Irido-corneal complexes (ICC's) (Connelly et al., '73) were removed from animals and placed in sterile Dulbecco phosphate buffered saline (Gibco) diluted to $80 \%$ (PBS-80) and containing $2 \mathrm{X}$ antibiotics. Lenses and other tissues adhering to the ICC's were removed in this solution and each complex was rinsed two additional times in PBS-80. Pituitaries were removed from newt heads after decapitation of the donors and careful rinsing of the oral surface with $70 \%$ ethanol and sterile distilled water. Before explantation, the glands were also cleaned and rinsed three times in PBS-80 containing antibiotics. Tissues were explanted to an organ culture environment similar to that used by Connelly et al. ('73), except that

\footnotetext{
'Supported by grants from The National Institutes of Health (HD 08150), Horace H. Rackham School of Graduate Studies and American Cancer Society Grant IN40.P.

${ }^{2}$ Address to which correspondence should be sent
} 
TABLE 1

Regenerative response of iris cultured in L-15(modified) for 21 days either alone or in direct contact with a newt pituitary

\begin{tabular}{|c|c|c|c|}
\hline & $\begin{array}{c}\text { No depigmentation } \\
\text { (Stages 1-2) }\end{array}$ & $\begin{array}{c}\text { Depigmentation } \\
\text { only } \\
\text { (Stages } 3 \cdot 5 \text { ) }\end{array}$ & $\begin{array}{c}\text { Depigmentation } \\
\text { and } \\
\text { lens formation } \\
\text { (Stages } 6.10)\end{array}$ \\
\hline With pituitary & $3 / 36$ & $4 / 36$ & $29 / 36$ \\
\hline Controls (no pituitary) & $2 / 8$ & $5 / 8$ & $1 / 8^{*}$ \\
\hline
\end{tabular}

In all tables the data are presented as the number of positive cases/total number of cultures in each group.

This table represents the results of three separate repetitions of the same experiment.

* In tables 1 3, numbers designated by an asterisk indicate cases in which only a limited number of lens fiber cells could be detected histologically.

TABLE 2

Regenerative response of iris cultured next to but not in direct contact with pituitary gland for 21 days in 1.15

\begin{tabular}{lccc}
\hline & No depigmentation & & \multicolumn{2}{c}{$\begin{array}{c}\text { Depigmentation } \\
\text { and } \\
\text { (Stages 1-2) }\end{array}$} & $\begin{array}{c}\text { Depigmentation } \\
\text { only } \\
\text { (Stages 3-5) }\end{array}$ & $\begin{array}{c}\text { lens formation } \\
\text { (Stages 6*10) }\end{array}$ \\
\hline With pituitary & $15 / 30$ & $13 / 30$ & $2 / 30^{*}$ \\
Controls (no pituitary) & $5 / 10$ & $5 / 10$ & $0 / 10$ \\
\hline
\end{tabular}

The results of the pituitary cultures are pooled data from two repetitions of the experiment.

For result of culturing iris in contact with pituitary see table 1 .

a detoxified Nuclepore filter was used as a supporting substrate and modified L-15 medium (Ortiz et al., '73) was used rather than Medium 199. The Nuclepore filters were rinsed in medium several times during a 24 -hour period before they were used. The culture medium consisted of $50 \%$ L- 15 medium, $40 \%$ sterile distilled water and $10 \%$ fetal calf serum. The antibiotic combination was that of Laskey ('70) and contained kanamycin, polymixin E, and fungizone. This combination provides contamination-free cultures in better than $90 \%$ of the cases. Medium was changed every third day.

To confirm earlier studies and to test the efficiency of L-15 medium in supporting lens regeneration in long term organ culture several groups of ICC's were cultured directly on top of pituitary glands with the mid-dorsal iris in direct contact with the gland. Control cultures consisted of ICC's cultured for 21 days without any other tissues in the dish. To determine if direct contact between the two organs is required, one group of ICC's was cultured next to, but not in contact with, the pituitary glands. Another group was cultured on top of pituitaries but separated from them by a 10-micron thick Nuclepore filter with 0.45-micron pores. Twenty-one days after ex- plantation all cultures were fixed and examined histologically. Regenerates were grouped as described before (Connelly et al., '73). To determine approximately when the presence of the pituitary gland is necessary for enhancement of regeneration in culture, ICC's were explanted, and 5,10 or 15 days later pituitary glands were added beneath the dorsal iris. Controls consisted of iris cultured alone or in contact with the pituitary for 21 days. Attempts were also made to explant iris with pituitary and then remove the glands at 5,10 or 15 days after explantation.

\section{RESULTS}

Table 1 shows the response of ICC's to prolonged culture in L-15 medium in the presence or absence of the pituitary. The medium and the new culture procedures allow for the production of lens regenerates of advanced morphology in $75-90 \%$ of the cases. This demonstrates the validity of the methods used. Table 1 also shows that the primary response of the irises cultured in the absence of the pituitary is the formation of depigmented masses without morphologically distinct lens regenerates. However, in some cases a small number of histologically identifiable lens fiber cells do differentiate in control cultures. Table 
TABLE 3

Regenerative response of iris cultured with pituitary but separated from it by a Nucleopore filter (in L-15 or Medium 199) for 21 days

\begin{tabular}{lccc}
\hline & No depigmentation \\
(Stages 1-2) & $3 / 10$ & $\begin{array}{c}\text { Depigmentation } \\
\text { only } \\
\text { (Stages 3-5) }\end{array}$ & $\begin{array}{c}\text { Depigmentation } \\
\text { and } \\
\text { lens formation } \\
\text { (Stages 6-10) }\end{array}$ \\
\hline Controls (no pituitary) & $1 / 8$ & $5 / 10$ & $2 / 10^{*}$ \\
Transfilter-newt pituitary & $6 / 8$ & $1 / 8^{*}$ \\
\hline
\end{tabular}

For result of culturing iris in contact with pituitary see table 1 .

TABLE 4

Effect of addition of pituitary gland to irido-corneal complexes 5,10 , or 15 days after initial explantation of the iris

\begin{tabular}{|c|c|c|c|}
\hline & $\begin{array}{c}\text { No depigmentation } \\
\text { (Stages 1-2) }\end{array}$ & $\begin{array}{c}\text { Depigmentation } \\
\text { only } \\
\text { (Stages 3-5) }\end{array}$ & $\begin{array}{c}\text { Depigmentation } \\
\text { and } \\
\text { lens formation } \\
\text { (Stages } 6-10 \text { ) }\end{array}$ \\
\hline Controls (no pituitary) & $2 / 14$ & $12 / 14$ & $0 / 14$ \\
\hline Day 5 & $0 / 24$ & $5 / 24$ & $19 / 24$ \\
\hline Day 10 & $3 / 25$ & $13 / 25$ & $9 / 25$ \\
\hline Day 15 & $8 / 22$ & $13 / 22$ & $1 / 22$ \\
\hline
\end{tabular}

The results from the pituitary addition cultures are pooled from three repetitions of the experiment.

For 21-day iris-pituitary combinations see table 1 .

2 shows that culturing the iris next to the pituitary but not in direct contact results in a marked reduction in the number of advanced lens regenerates formed when compared to cultures of iris plus pituitary. In those cases where histologically identifiable lens fiber cells are found the number of such cells is extremely small. Cultivation of the two organs separated by a Nuclepore filter also results in the formation of advanced regenerates in only a few cases (table 3). Unpublished experiments have also shown that although cultivation of a newt iris on top of a frog pituitary results in the formation of a high percentage of lens regenerates (Connelly et al., '73), interposition of a Nuclepore filter between these two organs also results in production of advanced regenerates in the same frequency reported here (Connelly, unpublished).

Finally, table 4 shows the results of adding pituitary glands to iris cultures at 5,10 or 15 days after initial explantation of the ICC's. These data show that the two organs need to be in contact from at least day 5 in order to produce numbers of advanced lens regenerates similar to those cultures which have had pituitary present from the first day. Addition of pituitary at day 10 results in some enhancement of regenerative activity, but there is still a marked reduction in the number of advanced regenerates produced, approaching levels found in irises cultured alone. Addition of the pituitary gland as late as day 15 results in the production of advanced lens regenerates in numbers similar to control groups which have been cultured 21 days without the gland. These results are a compilation of data from three repetitions of the experiment. Thus, it appears that the iris and the pituitary must be in direct contact with each other, and that this contact is most important during the first five to ten days in culture.

The experiment in which pituitaries were removed from ICC's 5,10 or 15 days after explantation gave ambiguous results since at 10 and 15 days, removal of the pituitary caused damage to the mid-dorsal pupillary margin. By ten days in culture a rather tight adherence between the two organs had developed and removal of the pituitary resulted in removal of pieces of iris as well. However, at five days the two organs were not as tightly adherent, and could be successfully separated. In the few such cultures we performed the number of lens regenerates produced by 21 days was comparable to control cultures which had never had pituitary glands.

\section{DISCUSSION}

The results of the experiments described above demonstrate: (1) that L-15 medium modified according to Ortiz et al. ('73), but 
containing Laskey's ('70) antibiotic-antimycotic mixture, is capable of supporting complete transformation of iris cells into lens cells in iris-pituitary combinations in organ culture; (2) that direct contact between the iris and the pituitary is required for enhancement of lens regeneration by the pituitary to occur in vitro; and (3) that the important interactions between the two organs occur during the first five to ten days after explantation.

It is not known how the pituitary gland enhances the production of advanced lens regenerates in culture. Hypophysectomy retards, but does not prohibit, lens regeneration in vivo (Schotte and Murphy, '53; Stone and Steinitz, '53; Connelly et al., '73). However, implantation of a pituitary gland into the anterior chamber of the newt eye can result in the induction of an accessory lens, even in the presence of the normal lens, if the pituitary and the iris come into close contact (Powell and Segil, '76). The greater the distance between the two organs, the lower the response of the iris. One possible interpretation of these results, in light of the results of organ culture experiments, is that in vivo the retina compensates for the loss of the pituitary, and that addition of a pituitary to the eye can result in an overabundance of some stimulatory factor if the iris and pituitary are close enough together. In vitro the presence of the pituitary is enough to compensate for the absence of the neural retina if the iris and the pituitary are in direct contact. There is no convincing evidence that pituitary hormones (or other hormones) act directly on cells engaged in regeneration. Vethamany-Globus and Liversage ('73) have reported an effect of prolactin, thyroxine, cortisol, and insulin on the production of cartilage by newt tail blastemas in vitro. In their experiments, blastemas already in the process of regeneration were cultured for only four days. In the system described in the present paper, irises are explanted before the regeneration process has begun, and are maintained for a prolonged period in culture. Experiments performed in this laboratory to test the efficiency of a combination of insulin, prolactin, thyroxine, and cortisol (doses similar to those of VethamanyGlobus) have not demonstrated any significant effect of this combination of hormones on the production of advanced lens regenerates by ICC's in vitro (Connelly, unpublished). Further studies using numerous other dose levels and combinations of these hormones are now in progress, and may help to determine if the hormonal components of the pituitary are involved in the enhancement of lens regeneration in culture. There is a possibility that the effect of the pituitary is due to a passive release of some substance(s) during the early days of culture. No direct evidence is available on this point, but it is known that preculturing the pituitary for four days prior to adding iris has no effect on the ability of the gland to enhance lens regeneration in vitro (Connelly, unpublished). It would be possible to cultivate newt pituitaries for much longer periods prior to adding iris tissue to the culture. This would presumably reduce the passive release of some substances and increase the production of prolactin and melanocyte-stimulating hormone by the pituitary as a result of removing the gland from negative hypothalamic control (Masur, '69). Such experiments have not been done.

There appear to be some differences in the ability of intact and dissociated iris epithelia to produce lens fibers in culture. Eguchi et al. ('74) have shown that lens crystallin-containing lentoids can be found in cell cultures of iris epithelia derived from a Japanese newt and grown in modified L-15 medium. In this laboratory, small lentoid bodies have been found among iris epithelial cells which have migrated out from iris explants even if the original iris explant remains in the dish (Connelly, unpublished). Unfortunately, there is no chemical confirmation of the presence of lens crystallins in these lentoids. There is currently no explanation for the ability of dissociated iris epithelial cells in culture to produce numerous crystallin-containing lentoid bodies while intact irises explanted in vitro or transplanted to various ectopic sties (Reyer et al., '73) fail to produce regenerates or large numbers of lens fiber cells.

Very little is known about intercellular communication in the iris relative to the control of the expression of potential for lens regeneration in intact iris in vivo or in vitro, or in dissociated iris epithelium. There is also very little known about the relationship between the number of cell divisions a formerly pigmented iris epithelial cell must go through before redifferentiating into a lens fiber cell, although Yamada et al. ('75) have worked out approximations of the number of cell cycles occurring between the onset of dedifferentiation and redifferentiation in the iris in vivo. 
It may be that an iris epithelial cell must pass through a prescribed number of cell division cycles before redifferentiating into a lens fiber cell (Yamada, personal communication). In vitro, the cells of an intact iris may not be able to proceed through the requisite number of cell division cycles before the adverse effects of long term organ culture disrupt the ability of the epithelial cells to express their potential to form lens. Addition of a pituitary (Connelly et al., '73) or neural retinal tissue (Yamada et al., '73) to the cultures may enhance the ability of the cells of an intact iris to proceed through the required number of cell division cycles and thus, enhance the regenerative activities of intact irises in organ culture. Dissociation of the iris epithelium may free the cells from some inhibition which allows them to proceed through the required number of cell cycles. The results of Connelly et al. ('73) showed that there is a significant effect of hypophysectomy on mitotic activity in the dorsal iris cells in the first phases of regeneration in vivo, and that prolonged hypophysectomy results in the retardation of the whole regeneration process. Unpublished studies in this laboratory have shown that irises cultured with pituitary glands have a higher thymidine labeling index 10 to 12 days after explantation than do irises cultured alone. Jauker and Yamada ('73) also reported reduced thymidine incorporation by iris epithelium after ten days in culture. Thus, the possibility exists that one of the ways the pituitary gland enhances lens regeneration in culture is through an effect on the mitotic activity of the iris epithelial cells. The results of the experiments in which a pituitary gland was added at later times after explantation of the iris lend some circumstantial support to this hypothesis, since addition of the glands after the first ten days in culture did not result in significant enhancement of regeneration. Likewise, removal of the pituitary after only five days in contact with the iris resulted in regenerative activities similar to irises cultured alone. Thus, it appears that the important interactions between the two organs take place from days 5 to 10 after explantation. This corresponds to the period of most intense mitotic activity in vivo. It has not been determined whether there might also be some effect on the production of a significant population of fully depigmented cells or on some other dedifferentiative event(s). Since depigmentation may be controlled through the cell cycle (Yamada, personal communication) it would be difficult to distinguish a direct effect of the pituitary on the depigmentation process from some effect on the cell cycle. Irises explanted after stage IV of regeneration has been attained have little trouble proceeding to stages where advanced lens fiber formation takes place (Eisenberg-Zalik and Scott, '69; Eguchi, '66). It, therefore, appears that it is not necessarily the redifferentiative phases of regeneration which are the most sensitive to the presence of the pituitary or neural retina in vitro. It is certainly possible that covert differentiative events occur during the time when the pituitary is required, and that these events may not be directly related to mitotic activity in the iris at all. Further work is needed to distinguish between these possibilities.

It is impossible to say that no contact is established between iris and pituitary gland in the Nuclepore transfilter experiments described above. It is known that cell processes can traverse the filter through the pores (Hay and Meier, '76). In any case, the contact between the two organs is greatly reduced by interposition of the filter barrier. The necessity for direct contact is emphasized also by the experiments in which iris and pituitary were cultured side by side. Experiments are currently in progress to examine at both the light and electron microscopic levels, the interface between the iris and the pituitary during a 21-day cultivation period to determine the extent and nature of the contact which develops between them. The results of the experiments reported above and those of Powell and Segil ('76) demonstrate that the pituitary can play an important role in the transformation of iris epithelial cells into lens fiber cells during Wolffiian lens regeneration both in vivo and in vitro if the two organs come into close enough contact.

\section{ACKNOWLEDGMENTS}

The author wishes to thank Ms. Elizabeth K. Ashcraft for her excellent technical assistance during the performance of this study, and to Doctors Tuneo Yamada and Jose R. Ortiz for critically reviewing the manuscript. Thanks are also due Ms. Roberta Castro for typing the manuscript.

\section{LITERATURE CITED}

Connelly, T. G., J. R. Ortiz and T. Yamada 1973 Influence of the pituitary on Wolffitan lens regeneration. Devel. Biol., 31: 401-415. 
Eguchi, G. 1966 In vitro analyses of Wolffian lens regeneration: differentiation of the regenerating lens rudiment of the newt Triturus pyrrhogaster. Embryol., 9:246266.

Eguchi, G., S. I. Abe and K. Watanabe 1974 Differentiation of lens-like structures from newt iris epithelial cells in vitro. Proc. Nat'l. Acad. Sci. (U.S.A.), 71: 5052-5056.

Eisenberg-Zalik, S., and V. Scott 1969 In vitrodevelopment of the regenerating lens. Devel. Biol., 19: 368-379.

Hay, E. D., and S. Meier 1976 Stimulation of corneal differentiation by interaction between cell surface and extracellular matrix. II. Further studies on the nature of transfilter "induction." Devel. Biol., 52: 141-157.

Jauker F, and T. Yamada 1973 Progressive alteration in the pattern of nucleic acid metabolism in the newt iris in cultivation. J. Exp. Zool., 183: 145-152.

Laskey, R. A. 1970 The use of antibiotics in the preparation of amphibian cell cultures from highly contaminated material. J. Cell Sci., 7: 653.659.

Masur, S. K. 1969 Fine structure of the autotransplanted pituitary in the red eft, Notophthalmus viridescens. Gen. Comp. Endocrinol., 12: 12-32.

Ortiz, J. R., T. Yamada and A. W. Hsie 1973 Induction of the stellate configuration in cultured iris epithelial cells by adenosine $3^{\prime}: 5^{\prime}$-cyclic monophosphate. Proc. Nat'l. Acad. Sci. (U.S.A.), 70: 2286-2290.

Powell, J. A., and N. Segil 1976 Secondary lens formation caused by implantation of pituitary into the eyes of the newt, Notophthalmus. Devel. Biol., 52: 128-140.

Reyer, R. W., R. A. Woolfitt and L. T. Withersty 1973 Stimulation of lens regeneration from newt dorsal iris when implanted into the blastema of a regenerating limb. Devel. Biol., 32: 258.281.

Rounds, D. E. 1973 Adult amphibian tissues and leukocytes. In: Tissue Culture: Methods and Applications. P. F. Kruse, Jr. and M. K. Patterson, Jr., eds. Academic Press, New York, pp. 129-133.

Schotte, O. E., and G. W. Murphy 1953 Regeneration of the lens in the absence of the pituitary in the adult newt (Triturus viridescens). J. Morph., 93: 447-464.

Stone, L. S., and H. Steinitz 1953 Effects of hypophyysectomy and thyroidectomy on lens and retina regeneration in the adult newt, Triturus V. viridescens. J. Exp. Zool., 124: 469-504.

Vethamany-Globus, S, and R. A. Liversage 1973 In vitro studies of the influence of hormones on tail regeneration in adult Diemictylus viridescens. J. Embryol. Exp. Morphol., 30: 397-413.

Yamada, T., D. H. Reese and D. S. McDevitt 1973 Transformation of iris into lens in vitroand its dependence on neural retina. Differentiation, $1: 65-82$

Yamada, T, M. Roesel and J. J. Beuchamp 1975 Cell cycle parameters in dedifferentiating iris epithelial cells. J. Embryol. Exp. Morphol., 34: 497-510. 